九州大学学術情報リポジトリ

Kyushu University Institutional Repository

Combination of hepatocellular markers is useful for prognostication in gastric hepatoid adenocarcinoma

\title{
長田，美佳子
}

https://doi.org/10.15017/1456004

出版情報：九州大学，2014，博士（医学），課程博士 バージョン：

権利関係：やむを得ない事由により本文ファイル非公開（2） 
Elsevier Editorial System(tm) for Human Pathology

Manuscript Draft

Manuscript Number: YHUPA-D-13-00807R1

Title: Combination of hepatocellular markers is useful for prognostication in gastric hepatoid adenocarcinoma

Article Type: Original Article

Keywords: hepatoid adenocarcinoma; solid; SALL4; PLUNC; HepPar-1

Corresponding Author: Dr. Yoshinao Oda, MD, PhD

Corresponding Author's Institution: Kyushu University, Graduate School of Med. Sciences

First Author: Mikako Osada

Order of Authors: Mikako Osada; Shinichi Aishima; Minako Hirahashi; Nobuyoshi Takizawa; Shunsuke Takahashi; Kazuhiko Nakamura; Masao Tanaka; Yoshihiko Maehara; Ryoichi Takayanagi; Yoshinao Oda, MD, PhD

Abstract: Hepatoid or AFP-producing adenocarcinoma of stomach growing in a solid pattern are highly aggressive tumors. It is difficult to detect hepatoid differentiation solely on the basis of findings from hematoxylin and eosin stainings, especially in small biopsy specimens. Gastric adenocarcinomas with hepatoid differentiation should be distinguished from solid type gastric adenocarcinoma, because of their different biological behavior. We immunohistochemically analyzed hepatocellular markers (AFP, Glypican-3, HepPar-1) and possible markers of gastric hepatoid adenocarcinoma (SALL4 and PLUNC) to detect hepatoid differentiation in 45 gastric hepatoid adenocarcinoma and 47 non-hepatoid solid type poorly differentiated adenocarcinoma. There were a higher incidence of vascular invasion $(\mathrm{P}=0.0055)$ and distant metastasis $(\mathrm{P}=0.0458)$ in hepatoid adenocarcinoma than in non-hepatoid adenocarcinoma. AFP, SALL4, HepPar-1, and Glypican-3 were significantly higher in hepatoid adenocarcinoma than in non-hepatoid adenocarcinoma. All five markers were positive in both the hepatoid/solid and the tubular component. In hepatoid adenocarcinoma, the frequency of distant metastasis was significantly higher in SALL4-negative cases than in SALL4-positive cases ( $\mathrm{P}=0.0381)$. HepPar-1 was associated with liver metastasis $(\mathrm{P}=0.0452)$. PLUNC was correlated with lymph node metastasis $(\mathrm{P}=0.0375)$. There was a significant difference in the survival rate between HepPar-1positive and HepPar-1-negative groups ( $\mathrm{P}=0.0437)$. The co-expression of PLUNC and SALL4 and the other co-expression of HepPar- 1 and PLUNC were associated with poorer prognosis $(\mathrm{p}=0.0181$ and $\mathrm{p}=0.0443$, respectively). AFP, SALL4, HepPar-1 and Glypican-3 are useful for the detection of hepatoid differentiation. A combination of PLUNC, HepPar-1 and SALL4 could be a reliable prognosic indicator in hepatoid adenocarcinoma of the stomach. 
Dr. Ricardo. V. Lloyd

Editor-in-Chief

Human Pathology

January 21, 2014

Dear Dr. Ricardo V. Lloyd

Thank you very much for your letter and the reviewers' comments on our manuscript entitled "Combination of hepatocellular markers is useful for prognostication in gastric hepatoid adenocarcinoma" by Mikako Osada et al. The comments offered by the reviewers were very helpful and we have revised our manuscript to incorporate your suggestions and the reviewers' suggestions as follows:

\section{Comments and our replies}

\section{Editors' comments:}

1. Please reduce the length of the main text by $15 \%$.

Reply: We reduced the introduction in main text p.4, line 14-15, the Material and methods in paragraph 2.1 p.6, line 4-6, 8-10, the Results in paragraph 3.1 p.9, line 17-18, p.10, line 1, in paragraph 3.2 p.10, line 9-10, in paragraph 3.4 p.12, line 6 and 10-11 and the Discussion p.13, line 8-10, p.15. line 14-18 and p.16, line 6-9 according to your comment. We added the explanation in paragraph 2.1 p.6, line 13 of Material and methods and in the Discussion p.16. line 3. We rewrote the explanation in paragraph 2.1 p.6 ,line 15-16 of the Material and methods and the explanation in Table 1. Moreover, we permutate photos in Figure 2 and rewrote the explanation in paragraph 3.3 of the Results and Figure legends.

2. Please format the abstract per our journal style as a single paragraph without section headers.

Reply: We rewrote the abstract per your journal style and changed the abstract p.2, line2,3.

3. In table 4, please check pathologic T stage vs PLUNC. Are the values for pT1-pT2 
and pT3-pT4 reversed?

Reply: We rewrote the values for pT1-pT2 and pT3-pT4 vs PLUNC in table 4.

\section{Reviewers' comments:}

\section{Reviewer \#2:}

1. Although the authors agree that the differentiation of hepatoid and non-hepatoid tumors may be challenging, they apparently did not have this problem since they could assign their solid/tubular tumors into one or the other category based on hematoxylin and eosin staining. It would be interesting to know if they reclassified some of the cases when the immunohistochemical stains became available. Also, we have to assume that tumors were homogenously hepatoid and non-hepatoid. Were there tumors that showed both features? If this is the case, how did tumor showing both hepatoid and non-hepatoid areas stain? Were there areas of common intestinal or diffuse gastric cancer associated with the hepatoid tumors? If this is the case, how did these areas stain? What was the background non-neoplastic mucosa?

Reply: We defined hepatoid adenocarcinoma as a tumor containing components characterized by morphological similarities to hepatocellular carcinomas despite the percentage of hepatoid components. Gastric hepatoid adenocarcinoma also frequently co-existed with a tubular adenocarcinoma component, but didn't contain diffuse gastric cancer. Non-hepatoid adenocarcinoma were defined as solid-type poorly differentiated adenocarcinoma without hepatoid component, prominent lymphoid stroma and neuroendocrine component in our study. In the background non-neoplastic mucosa, intestinal metaplasia was observed in $71 \%$ of gastric hepatoid adenocarcinoma.

2. In paragraph 2.3 of Material and methods, the authors state that a case was considered positive "when any cancer cells were stained". What does any mean, one, two, how many? Is it possible to get a sense of the positive staining area: percentage of tumor staining, focal, patchy, diffuse, etc? It is crucial to define these terms and illustrate what they mean.

Reply: We rewrote the explanation in paragraph 2.3 of Material and methods, and 
showed that a case was considered positive " when cancer cells were focally stained".

3. In the results, on paragraph 3.2, they indicate that the markers were focally positive not only in the solid/hepatoid components but also in the tubular areas. Again, what does focally mean? If "any" tumor cell is considered positive and if cases stained "focally," do they think this information is applicable to biopsy specimens?

Reply: We deleted "focally" in the results on paragraph 3.2. If cases stained focally, we think this information is appliciable to biopsy specimens.

4. The authors excluded cases of solid carcinomas with abundant lymphoid infiltrates and EBER-positive cases. Actually, it would be quite interesting to know whether some of these markers are present in "any" cell or focally in these tumors since they are generally not confused with hepatoid differentiation and would be a good comparison group for tumors with a solid growth pattern.

Reply: We examined immunohistochemical staining in solid areas using five antibodies in 10 cases of solid carcinomas with abundant lymphoid infiltrates and EBER-positive cases and 10 cases of neuroendocrine tumors. In solid carcinomas with lymphoid stroma, two cases were positive for HepPar-1 and one case was positive for Glypican-3. In neuroendocrine tumors, one case was positive for HepPar-1 and two cases were positive for both Glypican-3 and SALL4. No cases were positive for AFP in both groups.

5. Finally, does the percentage of tumor staining for these markers influence the prognosis? Do tumors that show an occasional positive cell have the same prognosis as those that have patchy or diffuse staining? If this is the case, it would support the significance of even finding one or few positive cells. However this needs to be analyzed.

Reply: The percentage of tumor staining for these markers didn't influence the prognosis in our study. Tumors that show an occasional positive cell have the same prognosis as these that have patchy or diffuse staining. We added the explanation in 
the discussion p.16. line 3 .

We thank you for your very helpful suggestions which have improved our report. Your kind consideration of this revision for publication in Human Pathology would be appreciated.

Sincerely yours,

Yoshinao Oda, M.D. PhD.

Mikako Osada, M.D. 
Combination of hepatocellular markers is useful for prognostication in gastric hepatoid adenocarcinoma

Mikako Osada ${ }^{1}$, Shinichi Aishima ${ }^{1}$, Minako Hirahashi ${ }^{1}$, Nobuyoshi Takizawa ${ }^{1}$, Shunsuke Takahashi ${ }^{1}$, Kazuhiko Nakamura ${ }^{2}$, Masao Tanaka ${ }^{3}$, Yoshihiko Maehara ${ }^{4}$, Ryoichi Takayanagi ${ }^{2}$, Yoshinao Oda ${ }^{1}$.

${ }^{1}$ Department of Anatomic Pathology, Graduate School of Medical Sciences, Kyushu University, Higashi-ku, Fukuoka, Japan

${ }^{2}$ Department of Medicine and Bioregulatory Science, Graduate School of Medical Sciences, Kyushu University, Higashi-ku, Fukuoka, Japan

${ }^{3}$ Department of Surgery and Oncology, Graduate School of Medical Sciences, Kyushu University, Higashi-ku, Fukuoka, Japan

${ }^{4}$ Department of Surgery and Medical Science, Graduate School of Medical Sciences, Kyushu University, Higashi-ku, Fukuoka, Japan

Corresponding author: Yoshinao Oda, Department of Anatomic Pathology, Pathological Sciences, Graduate School of Medical Sciences, Kyushu University, Maidashi 3-1-1, Higashi-ku, Fukuoka 812-8582, Japan

E-mail: oda@surgpath.med.kyushu-u.ac.jp

Tel: +81-92-642-6061, FAX: +81-92-642-5968

Running title: Hepatoid differentiation of gastric adenocarcinoma

\section{Disclosure/conflict of interest}

The authors declare that there are no conflicts of interest to disclose. 


\section{Abstract}

Hepatoid or AFP-producing adenocarcinoma of stomach growing in a solid pattern are highly aggressive tumors. It is difficult to detect hepatoid differentiation solely on the basis of findings from hematoxylin and eosin stainings, especially in small biopsy specimens. Gastric adenocarcinomas with hepatoid differentiation should be distinguished from solid type gastric adenocarcinoma, because of their different biological behavior. We immunohistochemically analyzed hepatocellular markers (AFP, Glypican-3, HepPar-1) and possible markers of gastric hepatoid adenocarcinoma (SALL4 and PLUNC) to detect hepatoid differentiation in 45 gastric hepatoid adenocarcinoma and 47 non-hepatoid solid type poorly differentiated adenocarcinoma. There were a higher incidence of vascular invasion $(\mathrm{P}=0.0055)$ and distant metastasis $(\mathrm{P}=0.0458)$ in hepatoid adenocarcinoma than in non-hepatoid adenocarcinoma. AFP, SALL4, HepPar-1, and Glypican-3 were significantly higher in hepatoid adenocarcinoma than in non-hepatoid adenocarcinoma. All five markers were positive in both the hepatoid/solid and the tubular component. In hepatoid adenocarcinoma, the frequency of distant metastasis was significantly higher in SALL4-negative cases than in SALL4-positive cases $(\mathrm{P}=0.0381)$. HepPar-1 was associated with liver metastasis $(\mathrm{P}=0.0452)$. PLUNC was correlated with lymph node metastasis 
Osada M, et al. 3

$(\mathrm{P}=0.0375)$. There was a significant difference in the survival rate between HepPar-1-positive and HepPar-1-negative groups $(\mathrm{P}=0.0437)$. The co-expression of PLUNC and SALL4 and the other co-expression of HepPar-1 and PLUNC were associated with poorer prognosis $(\mathrm{p}=0.0181$ and $\mathrm{p}=0.0443$, respectively). AFP, SALL4, HepPar-1 and Glypican-3 are useful for the detection of hepatoid differentiation. A combination of PLUNC, HepPar-1 and SALL4 could be a reliable prognosic indicator in hepatoid adenocarcinoma of the stomach.

Key words: hepatoid adenocarcinoma; solid; SALL4; PLUNC; HepPar-1 


\section{Introduction}

Hepatoid adenocarcinoma is an extrahepatic tumor characterized by morphological similarities to hepatocellular carcinomas [1]. It often produces $\alpha$-fetoprotein (AFP) and shows aggressive features of extensive vascular invasion and frequent liver metastases [1-3]. The stomach is the organ in which hepatoid adenocarcinoma has most commonly been identified. The histological features of gastric hepatoid adenocarcinoma typically consist of a tubular adenocarcinoma component and solid growth of the carcinoma component, the latter of which is similar to a feature of hepatocellular carcinomas [4,5]. Thus, solid type gastric adenocarcinoma has the potential to contain an area of hepatoid features or of an AFP-producing component. Because gastric hepatoid adenocarcinoma is thought to be an aggressive tumor, we should detect hepatoid differentiation when we encounter gastric adenocarcinoma growing in a solid pattern. Although hepatoid adenocarcinoma is morphologically defined by hematoxylin and eosin (HE) staining, hepatoid differentiation and definite diagnosis are difficult to achieve solely on the basis of histological findings, especially in small biopsy specimens. Further immunohistochemical staining is necessary for differential diagnosis.

Hepatocellular differentiation can be detected by multiple immunohistochemical 
markers. Oncofetal proteins, such as AFP and Glypican-3, are reliable diagnostic markers for yolk sac tumors, hepatocellular carcinoma, and a special subgroup of gastric carcinoma that includes AFP-producing type, hepatoid type, and fetal phenotype of gastric carcinoma [6-11]. Hepatocyte paraffin 1 (HepPar-1) is a monoclonal antibody specific for normal and neoplastic hepatocytes [12]. HepPar-1 was expressed in hepatoid components and tubular components in gastric hepatoid adenocarcinoma [13]. Sal-like protein 4 (SALL4) is a zinc finger transcription factor that plays a role in maintaining self-renewal and pluripotency in embryonic stem cells, and has been used as a marker of germ cell tumor and AFP-producing gastric carcinoma $[14,15]$. The palate, lung, and nasal epithelium carcinoma-associated protein (PLUNC) is also expressed in gastric hepatoid adenocarcinoma $[16,17]$. However, the prognostic value of the expression of these proteins in gastric carcinoma has not been fully investigated.

We compared the immunohistochemical expression of five markers (AFP, SALL4, HepPar-1, Glypican-3, and PLUNC) between hepatoid adenocarcinoma and non-hepatoid adenocarcinoma, and examined the prognostic implications.

\section{Materials and Methods}




\subsection{Case selection}

Solid type poorly differentiated adenocarcinoma is composed of neoplastic cells growing in a solid or sheet-like pattern and has a well-defined boundary $[18,19]$. Hepatoid adenocarcinoma was morphologically defined as a tumor composed of large polygonal eosinophilic hepatocyte-like neoplastic cells in a sheet-like pattern based on the World Health Organization system [20]. AFP production was not needed to define hepatoid adenocarcinoma. Non-hepatoid adenocarcinoma was defined as a solid type poorly differentiated adenocarcinoma without prominent lymphoid stroma associated with Epstein-Barr infection and components of neuroendocrine tumor. We collected 45 cases of hepatoid adenocarcinoma with hepatocellular morphology and 47 cases of non-hepatoid solid type poorly differentiated adenocarcinoma in this study. Lymph-node metastasis was assessed in 90 of 92 cases. Distant (liver) metastasis was assessed in 85 of 92 cases. AFP-producing gastric carcinoma was defined if the serum AFP was elevated and/or the results of immunohistochemical staining for AFP were positive. These samples were histologically diagnosed at the Department of Anatomic Pathology of Kyushu University and its affiliated hospitals between 1979 and 2013. All patients had undergone curative resection, without preoperative chemotherapy or radiation therapy preoperatively. The research protocol was approved by the Kyushu 
University Medical human investigation committee (IRB\#25-213).

\subsection{Immunohistochemical Staining}

Immunohistochemical staining was performed using mouse monoclonal antibodies against HepPar-1 (clone OCH1E5; diluted at 1:200, Dako, Glostrup, Denmark), Glypican-3 (clone 1G12; diluted at 1:200, BioMosaics, Burlington, VT), SALL4 (clone 6E3; diluted at 1:1000, Abnova, Taipei, Taiwan), AFP (rabbit polyclonal; diluted at 1:400, Dako, Glostrup, Denmark), and PLUNC (goat polyclonal; diluted at 1:500, R\&D Systems, Abingdon, UK). Sections were cut to a thickness of $4 \mu \mathrm{m}$, deparaffinized in xylene, and dehydrated in ethanol. Endogenous peroxidase activity was blocked by 30 minutes of incubation with $0.3 \%$ hydrogen peroxidase in absolute methanol. Antigens were retrieved by microwave heating in citrate buffer ( $\mathrm{pH}$ 6.0) for 20 minutes (HepPar-1, Glypican-3, SALL4), and by using a pressure cooker at $125^{\circ}$ C(Decloaking Chamber; Biocare Medical, Walnut Creek, CA, USA) in a Reveal Decloaker (Biocare Medical) for 5 minutes (PLUNC). Antigen was not retrieved for AFP. Sections were incubated with a primary antibody overnight at $4{ }^{\circ} \mathrm{C}$, and subjected to the biotin-free, horseradish peroxidase enzyme-labelled polymer method (Envision+system; Dako). The labeled antigens were visualized by 
3,3'-diaminobenzidine tetrahydrochloride as chromogen. Finally, the sections were counterstained with hematoxylin.

\subsection{Immunohistochemical evaluation}

The results of cytoplasmic staining for AFP, PLUNC and HepPar-1, as well as membrane and cytoplasmic staining for Glypican-3 and nuclear staining for SALL4, were considered positive when cancer cells were focally stained. We defined positive or negative cases by the presence of positive tumor cells in a solid growth area. In cases with a tubular component, we also examined positive tumor cells in both the tubular and solid components.

\subsection{Statistical analysis}

Statistical analyses were performed using the $\mathrm{X}^{2}$ test, Fisher's exact test, and Student's t-test. Tumor-related survival was considered as the period of survival between surgery and the date of the last follow-up, or until death due to disease. The survival rate was estimated by using the Kaplan-Meier method. Survival data were available in 84 of 92 cases. All statistical analyses were performed using JMP Statistical Discovery Software (version 9.0.2; SAS Institute, Cary, NC, USA). Results 
were considered statistically significant if $\mathrm{P}<0.05$.

\section{Results}

\subsection{Clinical and histologic features of hepatoid adenocarcinoma and non-hepatoid}

\section{adenocarcinoma}

Clinicopathologic features were compared between hepatoid adenocarcinoma $(n=45)$ and non-hepatoid adenocarcinoma $(n=47)$ in Table 1. The results showed that age, sex, tumor size, pathologic T-stage, lymphatic permeation, and lymph node metastasis were similar between the two groups. There were higher incidences of vascular invasion $(\mathrm{P}=0.0055)$ and distant metastasis $(\mathrm{P}=0.0458)$ in hepatoid adenocarcinoma than in non-hepatoid adenocarcinoma.

Most hepatoid adenocarcinomas consisted of a hepatoid component and a tubular component (Figure 1A, 1B, 1C). In most hepatoid cases, hepatoid component accounts for more than $50 \%$ of the maximum cut surface, though in some cases it constitutes more than $90 \%$. Non-hepatoid solid type poorly differentiated adenocarcinoma cells having few stroma were found to grow in a solid or sheet-like pattern and to have a well-defined boundary (Figure 1D, 1E). 


\subsection{Immunohistochemical features of hepatoid adenocarcinoma and non-hepatoid}

\section{adenocarcinoma}

The proportions of positive AFP (80\%), SALL4 (47\%), HepPar-1 (69\%), and Glypican-3 (56\%) staining were significantly higher in hepatoid adenocarcinoma than in non-hepatoid solid adenocarcinoma. The positive rate of PLUNC in hepatoid cases (16\%) was lower than that in nonhepatoid solid type cases (26\%), but the difference was not statistically significant (Table 2).

Five markers (AFP, SALL4, HepPar-1, Glypican-3, and PLUNC) were positive not only in the solid/hepatoid component but also in the tubular adenocarcinoma area (Table 3). In hepatoid adenocarcinoma, the expression of AFP and Glypican-3 were significantly higher in the hepatoid area than in the tubular area $(\mathrm{P}=0.0007, \mathrm{P}=0.0057$, respectively). The positivity of HepPar-1 staining and that of PLUNC staining were lower in the solid/hepatoid area than in the tubular area, but not significantly. There was no significant difference in the positivity of these five markers between the solid area and the tubular area in non-hepatoid adenocarcinoma.

\subsection{Association between immunohistochemical findings and clinicopathologic}

\section{features in hepatoid adenocarcinoma and non-hepatoid adenocarcinoma}


The immunohistochemical results and the relationship between protein expression and clinicopathologic factors in hepatoid adenocarcinoma cases are shown in Table 4. The frequency of distant metastasis was significantly higher in SALL4-negative cases than in SALL4-positive cases (Figure 2A) $(\mathrm{P}=0.0381)$. HepPar-1 expression (Figure 2B) was associated with liver metastasis $(\mathrm{P}=0.0452)$. PLUNC expression (Figure 2C) was significantly correlated with lymph node metastasis $(\mathrm{P}=0.0375)$. We found no correlation between the expression of either AFP (Figure 2D) or Glypican-3 (Figure 2E) and any of the clinicopathologic characteristics in hepatoid adenocarcinoma cases.

In non-hepatoid adenocarcinoma cases, the expression of five markers were not significantly associated with any of the clinicopathologic characteristics or with prognosis (results not shown).

\subsection{Prognosis after surgery}

The prognosis of the patients with hepatoid adenocarcinoma seemed to be worse than those with non-hepatoid adenocarcinoma, but there was no statistical significance (Figure 3A).

The univariate analysis showed that the overall survival of hepatoid adenocarcinoma was associated with lymph node metastasis, distant metastasis, and 
liver metastasis. There was a significant difference in the survival rate between HepPar-1-positive cases and HepPar-1-negative cases in hepatoid adenocarcinoma $(\mathrm{P}=0.0437)$ (Figure 3B, Table 4).

When two markers were combined, the combination of PLUNC and SALL4 and the other combination of HepPar-1 and PLUNC were significantly associated with prognosis after surgery in hepatoid adenocarcinoma. The PLUNC+/SALL4+ group showed poor prognosis compared with the PLUNC-/SALL4+ group $(\mathrm{P}=0.0181)$ (Figure 3C). The HepPar-1+/PLUNC+ group showed a worse prognosis than the HepPar-1-/PLUNC - group (P=0.0443) (Figure 3D).

In non-hepatoid adenocarcinoma, there was no significant difference in the survival rate between positive and negative groups of each of these markers (results not shown).

\section{Discussion}

Our results show that AFP, Glypican-3, HepPar-1 and SALL4 differentiate hepatoid adenocarcinoma of the stomach from the solid undifferentiated type. The positive rates of AFP (80\%) and Glypican-3 (56\%) in our study were lower than those in 
previous studies $(92.1 \%$ and $100 \%$, respectively) in hepatoid adenocarcinoma [21]. On the other hand, the positive rate of HepPar-1 (69\%) was higher in our study than in previous studies $(38.1 \%)$. This indicates that hepatoid adenocarcinoma is similar to the characteristics of hepatocellular carcinomas and that HepPar-1 cannot help in the differential diagnosis between hepatoid adenocarcinoma and hepatocellular carcinoma. However, the patients with hepatoid adenocarcinoma showing HepPar-1 expression showed more frequent liver metastasis and had a significantly worse survival rate than patients without HepPar-1 expression, indicating that the presence of HepPar-1 can help to detect hepatoid differentiation and tumor aggressiveness. In non-hepatoid adenocarcinoma, the proportions of AFP (6\%) and Glypican-3 (9\%) expression were slightly higher than those in common gastric adenocarcinoma in previous studies $(0.8 \%$ and $3.4 \%$, respectively). It is more likely to have hepatoid differentiation potential in non-hepatoid solid type adenocarcinoma than in common gastric adenocarcinoma.

Our data show that the expression of PLUNC does not differ between hepatoid adenocarcinoma and non-hepatoid adenocarcinoma, though Sentani et al. [17] reported that PLUNC staining was found more frequently in gastric hepatoid adenocarcinoma than in other types (well and poorly differentiated adenocarcinoma) of gastric cancer. This result indicates that PLUNC is not an antibody specific to gastric hepatoid 
adenocarcinoma. However, all 7 PLUNC-positive cases showed vascular invasion and lymph node metastasis in hepatoid adenocarcinoma, suggesting that PLUNC expression may be a key factor indicating tumor malignant potential in hepatoid adenocarcinoma. These results suggest that hepatoid differentiation is difficult on the basis solely of findings from hematoxylin and eosin (HE) staining or from the expression of a single marker.

In the present study, all five markers were positive not only in the solid/hepatoid component but also in the tubular adenocarcinoma area. A comparison of immunohistochemical results between solid and tubular areas in hepatoid adenocarcinoma revealed AFP-positive tumor cells and Glypican-3-positive tumor cells with significant frequency in the solid area. Sentani et al. [17] reported that AFP-positive tumor cells were observed in hepatoid adenocarcinoma components, and Ushiku et al. [15] reported that Glypican-3 expression was observed more frequently in hepatoid than in glandular components, supporting our data. HepPar-1 staining and PLUNC staining were preferentially found in the tubular area. These data indicate that even a tubular adenocarcinoma can possess hepatoid differentiation, and that we should consider the possibility of hepatoid differentiation of tubular adenocarcinoma with a solid/hepatoid component. 
Co-expression of many markers such as PLUNC, HepPar-1, SALL4 and AFP may be a key factor in malignant potential when we encounter solid type poorly differentiated adenocarcinomas and suspected hepatoid adenocarcinomas. Moreover, the percentage of tumor staining for these markers didn't influence the prognosis in our study, supporting that significance of even finding one or few positive cells.

In summary, AFP, SALL4, HepPar-1, and Glypican-3 is useful when it is difficult to differentiate hepatoid adenocarcinoma from non-hepatoid solid type poorly differentiated adenocarcinoma. Co-expression of PLUNC and SALL4 and the other co-expression of HepPar-1 and PLUNC could help to indicate worse prognosis in hepatoid adenocarcinoma. 
Osada M, et al. 16

\section{Acknowledgments}

We thank all the technical staff of the Department of Pathology (Kyushu University) for their assistance. The English in this manuscript was revised by $\mathrm{KN}$ International. 


\section{References}

[1] Ishikura H, Fukasawa Y, Ogasawara K, Natori T, Tsukada Y, Aizawa M. An AFP-producing gastric carcinoma with features of hepatic differentiation. A case report. Cancer 1985;56:840-848.

[2] Ishikura H, Kishimoto T, Andachi H, Kakuta Y, Yoshiki T. Gastrointestinal hepatoid adenocarcinoma: venous permeation and mimicry of hepatocellular carcinoma, a report of four cases. Histopathology 1997;31:47-54.

[3] Nagai E, Ueyama T, Yao T, Tsuneyoshi M. Hepatoid adenocarcinoma of the stomach. A clinicopathologic and immunohistochemical analysis. Cancer $1993 ; 72: 1827-1835$.

[4] Ishikura H, Kirimoto K, Shamoto M, Miyamoto Y, Yamagiwa H, Itoh T, Aizawa M. Hepatoid adenocarcinomas of the stomach. An analysis of seven cases. Cancer 1986;58:119-126.

[5] Kumashiro Y, Yao T, Aishima S, et al. Hepatoid adenocarcinoma of the stomach: histogenesis and progression in association with intestinal phenotype. Hum Pathol. 2007;38:857-863.

[6] Kinjo T, Taniguchi H, Kushima R, et al. Histologic and immunohistochemical analyses of $\alpha$-fetoprotein-producing cancer of the stomach. Am J Surg Pathol 
2012;36:56-65.

[7] Gitlin D, Perricelli A, Gitlin GM. Synthesis of $\alpha$-fetoprotein by liver, yolk sac, and gastrointestinal tract of the human conceptus. Cancer Res. 1972;32:979-982.

[8] Yamauchi N, Watanabe A, Hishinuma M, et al. The glypican 3 oncofetal protein is a promising diagnostic marker for hepatocellular carcinoma. Mod Pathol 2005;18:1591-1598.

[9] Zynger DL, Dimov ND, Luan C, The BT, Yang XJ. Glypican 3: a novel marker in testicular germ cell tumors. Am J Surg Pathol 2006;30:1570-1575.

[10] Hishinuma M, Ohashi K-I, Yamauchi N, et al. Hepatocellular oncofetal protein, glypican 3 is a sensitive marker for $\alpha$-fetoprotein-producing gastric carcinoma. Histopathology 2006;49:479-486.

[11] Ushiku T, Uozaki H, Shinozaki A, et al. Glypican 3-expressing gastric carcinoma: Distinct subgroup unifying hepatoid, clear cell, and $\alpha$-fetoprotein-producing gastric carcinomas. Cancer Sci. 2009;100:626-632.

[12] Wennerberg AE, Nalesnik MA, Coleman WB. Hepatocyte Paraffin 1: A monoclonal antibody that reacts with hepatocytes and can be used for differential diagnosis of hepatic tumors. Am J Pathol 1993;143:1050-1054.

[13] Maitra A, Murakata LA, Albores-Saavedra J. Immunoreactivity for hepatocyte 
paraffin 1 antibody in hepatoid adenocarcinomas of the gastrointestinal tract. Anatomic Pathol 2001;115:689-694.

[14] Wang F, Liu A, Peng Y, et al. Diagnostic utility of SALL4 in extragonadal yolk sac tumors: an immunohistochemical study of 59 cases with comparison to placental-like alkaline phosphatase, alpha-fetoprotein, and glypican-3. Am J Surg Pathol 2009;33:1529-1539.

[15] Ushiku T, Shinozaki A, Shibahara J, Iwasaki Y, Tateishi Y, Funata N, Fukayama M. SALL4 represents fetal gut differentiation of gastric cancer, and is diagnostically useful in distinguishing hepatoid gastric carcinoma from hepatocellular carcinoma. Am J Surg Pathol 2010;34:533-540.

[16] Weston WM, LeClair EE, Trzyna W, et al. Differential display identification of plunk, a novel gene expressed in embryonic palate, nasal epithelium, and adult lung. $\mathrm{J}$ Biol Chem 1999;274:13698-13703.

[17] Sentani K, Oue N, Sakamoto N, Arihiro K, Aoyagi K, Sasaki H, Yasui W. Gene expression profiling with microarray and SAGE identifies PLUNC as a marker for hepatoid adenocarcinoma of the stomach. Mod pathol. 2008;21:464-475.

[18] Japanese Research Society for Gastric Cancer. Japanese Classification of Gastric Carcinoma, $14^{\text {th }}$ ed. Kanehara-Shuppan: Tokyo, 2010, 8-9,34-37. 
[19] Jiao Y, Sugai T, Habano W, Uesugi N, Takagane A, Nakamura S. Clinicopathological significance of loss of heterozygosity in intestinal- and solid-type gastric carcinomas: a comprehensive study using the crypt isolation technique. Mod Pathol. 2006;19:548-555.

[20] Bosman FT, Carneiro F, Hruban RH, Theise ND. WHO Classification of Tumors of the Digestive System. $4^{\text {th }}$ ed. IARC: Lyon 2010. 48-58.

[21] Jiann-Sheng S, Yu-Tso C, Ren-Ching W, Chun-Ying W, Shou-Wu L, Teng-Yu L. Clinicopathological characteristics in the differential diagnosis of hepatoid adenocarcinoma: A literature review. World J Gastroenterol 2013;19:321-327. 


\section{Figure legends}

\section{Figure 1}

A: Hepatoid adenocarcinoma consisted of tubular and solid components $(\times 40)$.

B: Tubular component $(\times 200)$. In the gastric mucosal layer, tubular proliferation of adenocarcinoma was found.

C: Solid component $(\times 400)$. Tumor cells having abundant eosinophilic cytoplasm were arranged in solid nests with sinusoid-like capillaries.

D: Non-hepatoid solid type poorly differentiated adenocarcinoma was composed of neoplastic cells growing in a solid or sheet-like pattern and had a well-defined boundary $(\times 100)$.

E: Non-hepatoid solid type poorly differentiated adenocarcinoma $(\times 400)$. The tumor cells have few stroma.

\section{Figure 2}

Immunohistochemical staining of SALL4 (A), HepPar-1 (B), PLUNC (C), AFP (D), and Glypican-3 (E) in the hepatoid component of hepatoid adenocarcinoma $(\times 200)$. Cytoplasmic staining for AFP, HepPar-1, and PLUNC; membrane and cytoplasmic staining for Glypican-3; and nuclear staining for SALL4 were observed. 
Osada M, et al. 22

\section{Figure 3}

A: The overall survival curves for hepatoid adenocarcinoma cases and non-hepatoid adenocarcinoma cases.

B: The overall survival curves for HepPar-1-positive cases and HepPar-1-negative cases in hepatoid adenocarcinoma.

C: The overall survival curves for the four groups by the combination of PLUNC and SALL4 in hepatoid adenocarcinoma.

D: The overall survival curves for the four groups by the combination of HepPar-1 and

PLUNC in hepatoid adenocarcinoma. 
Click here to download high resolution image
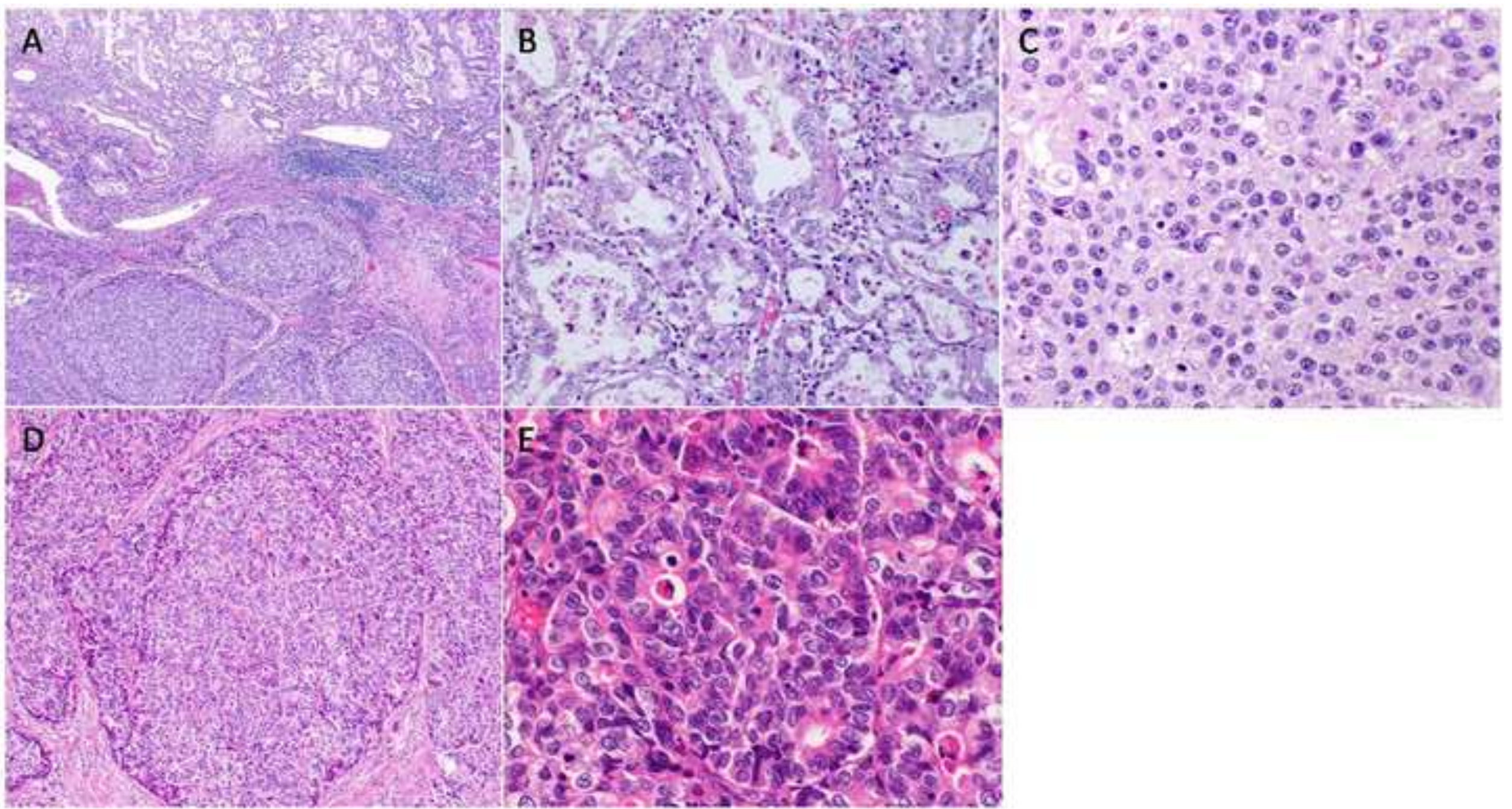

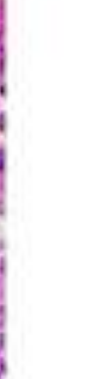


A

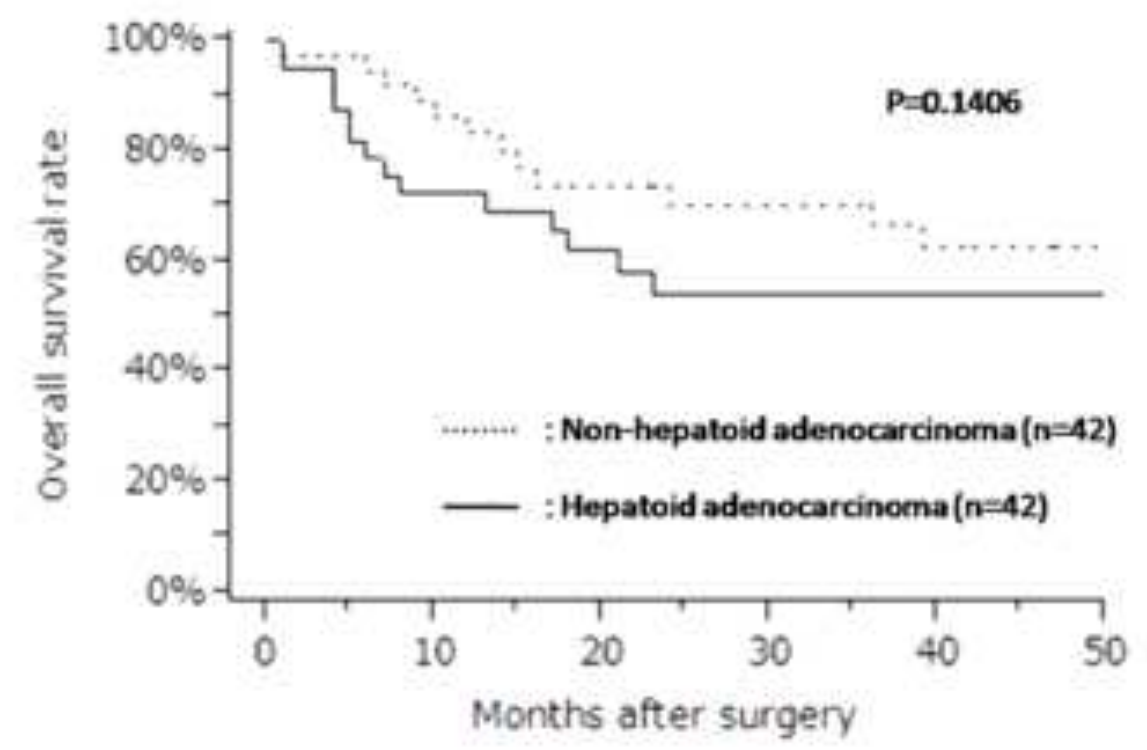

C

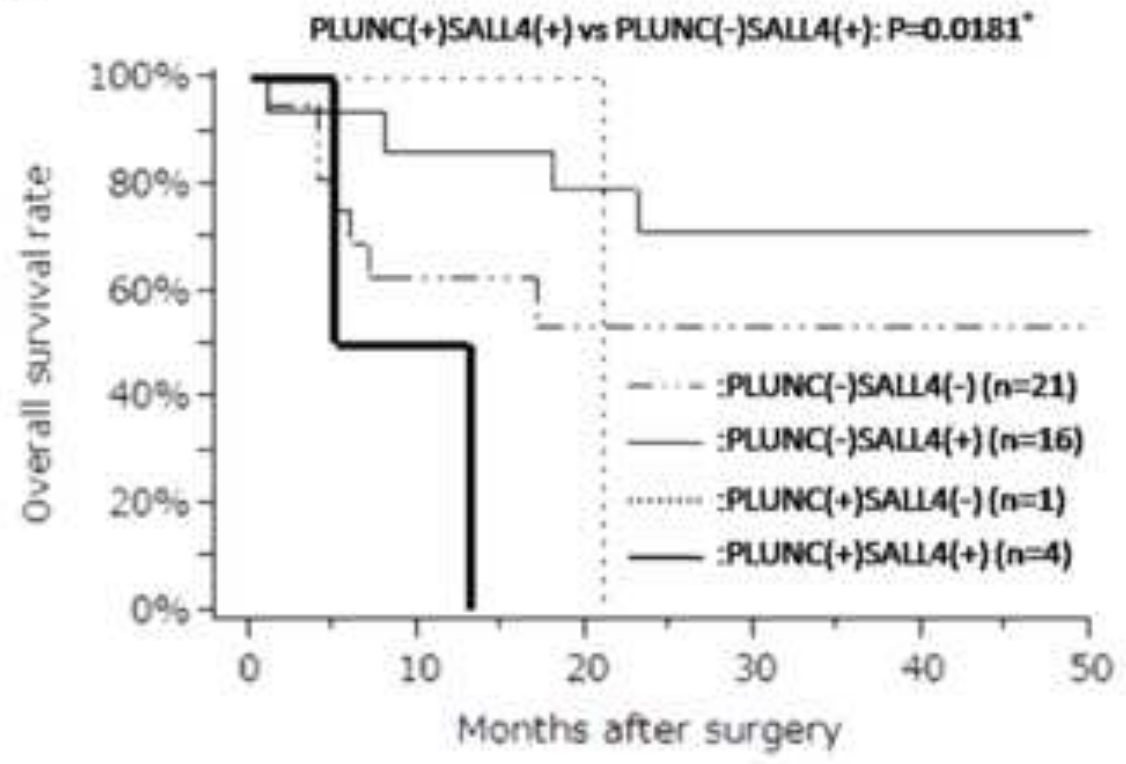

B

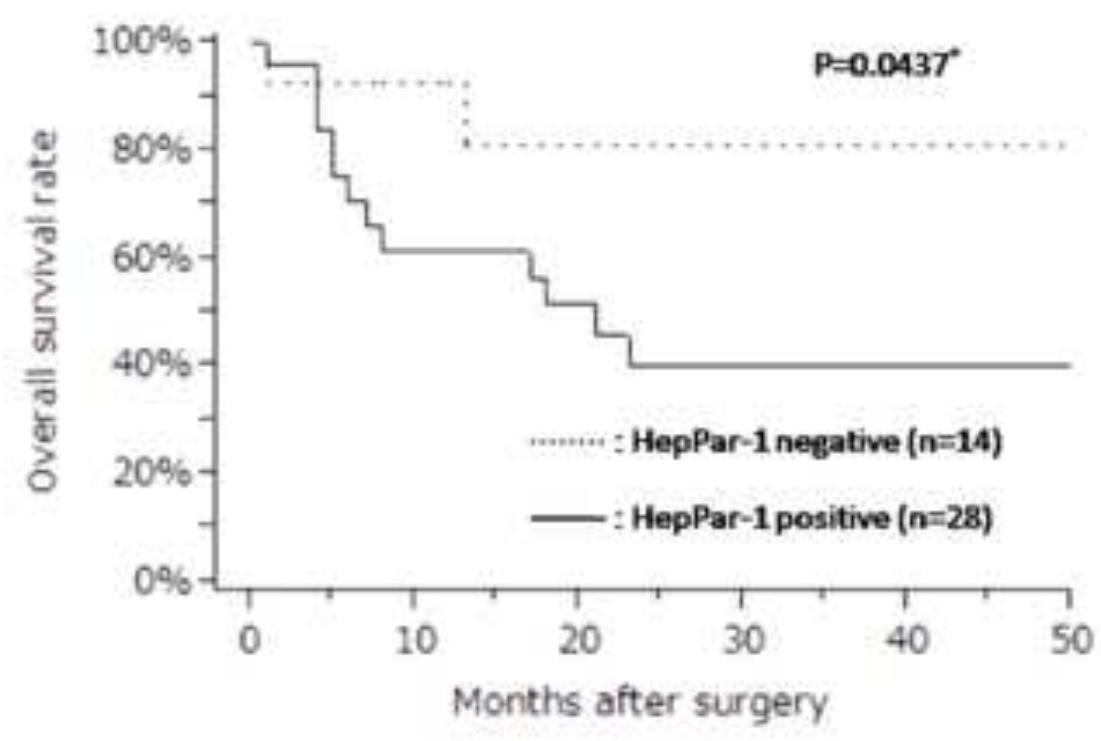

D

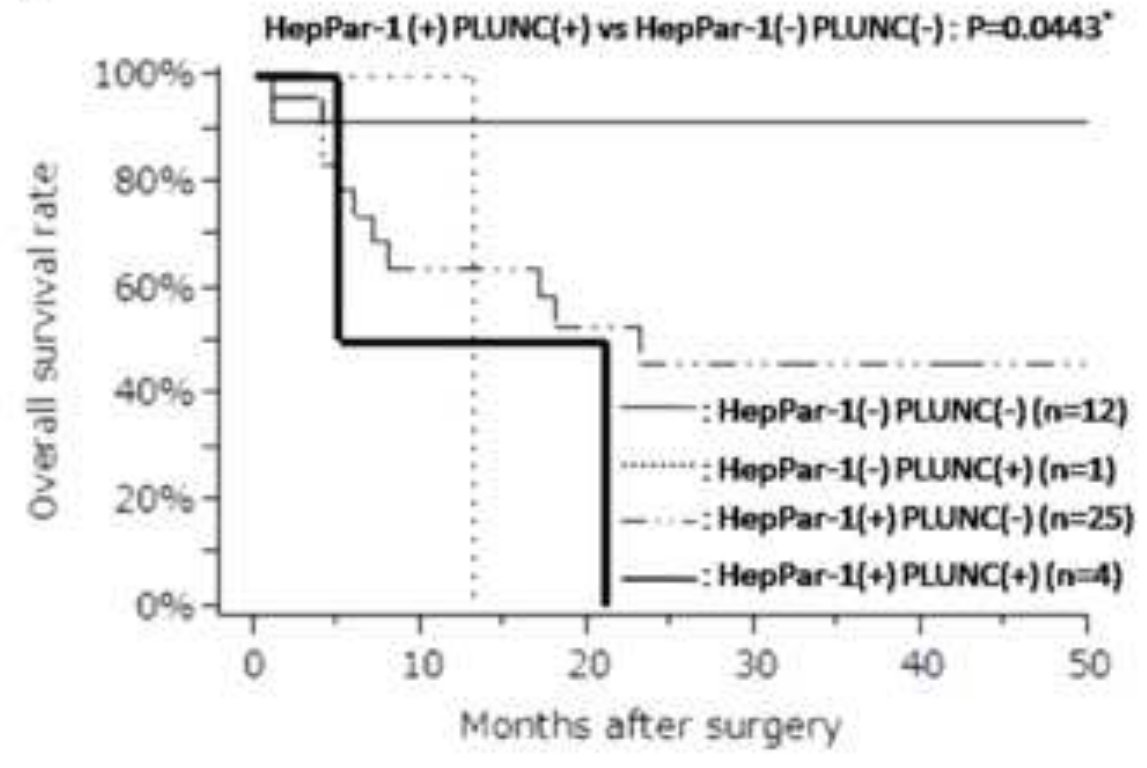


Table 1 Comparison of clinicopathologic characteristics between hepatoid adenocarcinoma and non-hepatoid adenocarcinoma

\begin{tabular}{|c|c|c|c|}
\hline & $\begin{array}{l}\text { hepatoid } \\
\mathrm{n}=45(\%)\end{array}$ & $\begin{array}{c}\text { non-hepatoid } \\
\text { n=47(\%) }\end{array}$ & p-value \\
\hline Age,y & & & 0.4015 \\
\hline$<70$ & $25(56)$ & $22(47)$ & \\
\hline$\geqq 70$ & $20(44)$ & $25(53)$ & \\
\hline Sex & & & 0.9246 \\
\hline Male & $32(71)$ & $14(30)$ & \\
\hline Female & 13(29) & $33(70)$ & \\
\hline Tumor size, $\mathrm{cm}$ & & & 0.297 \\
\hline$<6$ & $25(56)$ & $21(45)$ & \\
\hline$\geqq 6$ & $20(44)$ & $26(55)$ & \\
\hline Pathologic $\mathrm{T}$ stage & & & 0.4811 \\
\hline pT1-pT2 & $15(33)$ & $19(40)$ & \\
\hline pT3-pT4 & $30(67)$ & $28(60)$ & \\
\hline Lymphatic permeation & & & 0.0955 \\
\hline Present & $24(53)$ & $33(70)$ & \\
\hline Absent & $21(47)$ & $14(30)$ & \\
\hline Vascular invasion & & & $0.0055^{*}$ \\
\hline Present & $37(82)$ & $26(55)$ & \\
\hline Absent & $8(18)$ & $21(45)$ & \\
\hline Lymph node metastasis $\dagger$ & & & 0.1719 \\
\hline Present & $34(76)$ & $28(62)$ & \\
\hline Absent & $11(24)$ & $17(38)$ & \\
\hline Distant metastasis $\dagger$ & & & $0.0458^{*}$ \\
\hline Present & $18(40)$ & $8(20)$ & \\
\hline Absent & $27(60)$ & $32(80)$ & \\
\hline \multicolumn{4}{|l|}{ Liver metastasis $\dagger$} \\
\hline Present & $16(36)$ & $7(17)$ & 0.0584 \\
\hline Absent & $29(64)$ & $33(83)$ & \\
\hline
\end{tabular}

$\dagger$ In non-hepatoid adenocarcinoma, lymph node metastasis was assessed in 45 out of 47 cases and distant (liver) metastasis was assessed in 40 out of 47 cases. 

Table 2 Comparison of immunohistochemical expression between hepatoid adenocarcinoma and non-hepatoid adenocarcinoma

\begin{tabular}{rccc}
\hline & $\begin{array}{c}\text { hepatoid } \\
\mathrm{n}=45(\%)\end{array}$ & $\begin{array}{c}\text { non-hepatoid } \\
\mathrm{n}=47(\%)\end{array}$ & p-value \\
\hline AFP expression & & & \\
Positive & $36(80)$ & $3(6)$ & $<.0001^{*}$ \\
Negative & $9(20)$ & $44(94)$ & \\
SALL4 expression & & & \\
Positive & $21(47)$ & $3(6)$ & $<.0001^{*}$ \\
Negative & $24(53)$ & $44(94)$ & \\
HepPar-1 expression & & & \\
Positive & $31(69)$ & $11(23)$ & $<.0001^{*}$ \\
Negative & $14(31)$ & $36(77)$ & \\
Glypican-3 expression & & & \\
Positive & $25(56)$ & $4(9)$ & $<.0001^{*}$ \\
Negative & $20(44)$ & $43(91)$ & \\
PLUNC expression & & & \\
Positive & $7(16)$ & $12(26)$ & 0.2348 \\
Negative & $38(84)$ & $35(74)$ & \\
\hline & & & \\
\hline
\end{tabular}


Table 3 Immunohistochemical positive rate of AFP, SALL4, PLUNC, HepPar-1 and Glypican-3 of hepatoid adenocarcinoma and non-hepatoid adenocarcinoma in solid area and tubular area

\begin{tabular}{|c|c|c|c|c|c|c|c|c|c|c|c|c|c|c|c|}
\hline & \multicolumn{2}{|c|}{ AFP expression } & \multirow[b]{3}{*}{$\mathrm{p}$-value } & \multicolumn{2}{|c|}{ SALL4 expression } & \multirow[b]{3}{*}{$\mathrm{p}$-value } & \multicolumn{2}{|c|}{ HepPar-1 expression } & \multirow[b]{3}{*}{$\mathrm{p}$-value } & \multicolumn{2}{|c|}{ Glypican-3 expression } & \multirow[b]{3}{*}{ p-value } & \multicolumn{2}{|c|}{ PLUNC expression } & \multirow[b]{3}{*}{ p-value } \\
\hline & solid & tubular $\dagger$ & & solid & tubular $\dagger$ & & solid & tubular $\dagger$ & & solid & tubular $\dagger$ & & solid & tubular $\dagger$ & \\
\hline & area & area & & area & area & & area & area & & area & area & & area & area & \\
\hline $\begin{array}{l}\text { hepatoid } \\
\text { n=45 }\end{array}$ & $36(80 \%)$ & $11(41 \%)$ & $0.0007^{*}$ & $21(47 \%)$ & $9(33 \%)$ & 0.2666 & $31(69 \%)$ & $21(78 \%)$ & 0.4149 & $25(56 \%)$ & $6(22 \%)$ & $0.0057^{*}$ & $7(16 \%)$ & $6(22 \%)$ & 0.4765 \\
\hline $\begin{array}{c}\text { non-hepatoid } \\
\qquad \mathrm{n}=47\end{array}$ & $3(6 \%)$ & $1(4 \%)$ & 0.6483 & $2(4 \%)$ & $4(15 \%)$ & 0.0973 & $11(23 \%)$ & $8(31 \%)$ & 0.4922 & $4(9 \%)$ & $0(0 \%)$ & 0.126 & $12(26 \%)$ & $9(35 \%)$ & 0.4117 \\
\hline
\end{tabular}

$\dagger$ Tubular component was found in 27 out of 45 cases in hepatoid adenocarcinoma and 26 out of 47 cases in non-hepatoid adenocarcinoma. 
Table 4 Association between immunohistochemical expression and clinicopathologic characteristics in hepatoid adenocarcinoma cases

\begin{tabular}{|c|c|c|c|c|c|c|c|c|c|c|c|c|c|c|c|}
\hline & \multicolumn{2}{|c|}{ AFP } & \multirow[b]{3}{*}{$\mathrm{p}$-value } & \multicolumn{2}{|c|}{ SALL4 } & \multirow[b]{3}{*}{$\mathrm{p}$-value } & \multicolumn{2}{|c|}{ HepPar-1 } & \multirow[b]{3}{*}{$\mathrm{p}$-value } & \multicolumn{2}{|c|}{ Glypican-3 } & \multirow[b]{3}{*}{$\mathrm{p}$-value } & \multicolumn{2}{|c|}{ PLUNC } & \multirow[b]{3}{*}{$\mathrm{p}$-value } \\
\hline & positive & negative & & positive & negative & & positive & negative & & positive & negative & & positive & negative & \\
\hline & $\mathrm{n}=36(\%)$ & $\mathrm{n}=9(\%)$ & & $\mathrm{n}=21(\%)$ & $\mathrm{n}=24(\%)$ & & $\mathrm{n}=31(\%)$ & $\mathrm{n}=14(\%)$ & & $\mathrm{n}=25(\%)$ & $\mathrm{n}=20(\%)$ & & $\mathrm{n}=7(\%)$ & $\mathrm{n}=38(\%)$ & \\
\hline Age,y & & & 1 & & & 0.6885 & & & 0.6143 & & & 0.5915 & & & 0.9267 \\
\hline$<70$ & $20(56)$ & $5(56)$ & & $11(52)$ & $14(58)$ & & $18(58)$ & $7(50)$ & & $13(52)$ & $12(60)$ & & $4(57)$ & $21(55)$ & \\
\hline$\geqq 70$ & $16(44)$ & $4(44)$ & & $10(48)$ & $10(42)$ & & $13(42)$ & $7(50)$ & & $12(48)$ & $8(40)$ & & $3(43)$ & $17(45)$ & \\
\hline Sex & & & $0.0485^{*}$ & & & 0.173 & & & 0.1647 & & & 0.1413 & & & 0.3749 \\
\hline Male & $28(78)$ & $4(44)$ & & $17(81)$ & $15(62)$ & & $24(77)$ & $8(57)$ & & $20(80)$ & $12(60)$ & & $4(57)$ & $28(74)$ & \\
\hline Female & $8(22)$ & $5(56)$ & & $4(19)$ & $9(38)$ & & $7(23)$ & $6(43)$ & & $5(20)$ & $8(40)$ & & $3(43)$ & $10(26)$ & \\
\hline Tumor size,cm & & & 1 & & & 0.1606 & & & 0.6143 & & & 0.5023 & & & 0.4619 \\
\hline$<6$ & $20(56)$ & $5(56)$ & & $14(67)$ & $11(46)$ & & $18(58)$ & $7(50)$ & & $15(60)$ & $10(50)$ & & $3(43)$ & $22(58)$ & \\
\hline$\geqq 6$ & $16(44)$ & $4(44)$ & & $7(33)$ & $13(54)$ & & $13(42)$ & $7(50)$ & & $10(40)$ & $10(50)$ & & $4(57)$ & $16(42)$ & \\
\hline Pathologic T stage & & & 0.4292 & & & 0.2049 & & & 0.6488 & & & 0.6714 & & & 0.7712 \\
\hline pT1-pT2 & $13(36)$ & $2(22)$ & & $9(43)$ & $6(25)$ & & $11(35)$ & $4(29)$ & & $9(36)$ & $6(30)$ & & $2(29)$ & $13(34)$ & \\
\hline pT3-pT4 & $23(64)$ & $7(78)$ & & $12(57)$ & $18(75)$ & & $20(65)$ & $10(71)$ & & $16(64)$ & $14(70)$ & & $5(71)$ & $25(66)$ & \\
\hline Lymphatic permeation & & & 0.1003 & & & 0.0553 & & & 0.7633 & & & 0.1606 & & & 0.826 \\
\hline Present & $17(47)$ & $7(78)$ & & $8(38)$ & $16(67)$ & & $17(55)$ & $7(50)$ & & $11(44)$ & $13(65)$ & & $4(57)$ & $20(53)$ & \\
\hline Absent & $19(53)$ & $2(22)$ & & $13(62)$ & $8(33)$ & & $14(45)$ & $7(50)$ & & $14(56)$ & $7(35)$ & & $3(43)$ & $18(47)$ & \\
\hline Vascular invasion & & & 0.6966 & & & 0.5666 & & & 0.2031 & & & 0.6629 & & & 0.1806 \\
\hline Present & $30(83)$ & $7(78)$ & & $18(86)$ & 19(79) & & $27(87)$ & $10(71)$ & & $20(80)$ & $17(85)$ & & $7(100)$ & $30(79)$ & \\
\hline Absent & $6(17)$ & $2(22)$ & & $3(14)$ & $5(21)$ & & $4(13)$ & $4(29)$ & & $5(20)$ & $3(15)$ & & $0(0)$ & $8(21)$ & \\
\hline Lymph node & & & 0.4878 & & & 0.1943 & & & 0.2371 & & & 0.9382 & & & $0.0375^{*}$ \\
\hline
\end{tabular}




\begin{tabular}{|c|c|c|c|c|c|c|c|c|c|c|c|c|c|c|c|}
\hline Present & $28(78)$ & $6(67)$ & & $14(67)$ & $20(83)$ & & $25(81)$ & $9(64)$ & & $19(76)$ & $15(75)$ & & $7(100)$ & $27(71)$ & \\
\hline Absent & $8(22)$ & $3(33)$ & & $7(33)$ & $4(17)$ & & $6(19)$ & $5(36)$ & & $6(24)$ & $5(25)$ & & $0(0)$ & $11(29)$ & \\
\hline Distant metastasis & & & 0.2869 & & & $0.0381^{*}$ & & & 0.0875 & & & 1 & & & 0.4942 \\
\hline Present & $13(36)$ & $5(56)$ & & $5(24)$ & $13(54)$ & & $15(48)$ & $3(21)$ & & $10(40)$ & $8(40)$ & & $2(29)$ & $16(42)$ & \\
\hline Absent & $23(64)$ & $4(44)$ & & $16(76)$ & $11(46)$ & & $16(52)$ & $11(79)$ & & $15(60)$ & $12(60)$ & & $5(71)$ & $22(58)$ & \\
\hline Liver metastasis & & & 0.5334 & & & 0.1236 & & & $0.0452^{*}$ & & & 0.9445 & & & 0.6701 \\
\hline Present & $12(33)$ & $4(44)$ & & $5(24)$ & $11(46)$ & & $14(45)$ & $2(14)$ & & $9(36)$ & $7(35)$ & & $2(29)$ & $14(37)$ & \\
\hline Absent & $24(67)$ & $5(56)$ & & $16(76)$ & $13(54)$ & & $17(55)$ & $12(86)$ & & $16(64)$ & $13(65)$ & & $5(71)$ & $24(63)$ & \\
\hline 5-year survival, \% & 55 & 44 & 0.4783 & 63 & 47 & 0.2054 & 40 & 81 & $0.0437^{*}$ & 51 & 61 & 0.7884 & 0 & 61 & 0.0747 \\
\hline
\end{tabular}

